OPEN ACCESS

Edited by:

Martin G. Klotz,

Queens College (CUNY),

United States

Reviewed by:

Chiaki Kato,

Japan Agency for Marine-Earth

Science and Technology, Japan

Brett Pellock,

Providence College, United States

${ }^{*}$ Correspondence:

Fengping Wang

fengpingw@sjtu.edu.cn

Specialty section:

This article was submitted to

Aquatic Microbiology,

a section of the journal

Frontiers in Microbiology

Received: 06 March 2017

Accepted: 30 May 2017

Published: 15 June 2017

Citation:

Nawaz MZ, Jian H, He Y, Xiong L,

Xiao $X$ and Wang $F(2017)$

Genome-Wide Detection of Small

Regulatory RNAs in Deep-Sea

Bacterium Shewanella piezotolerans

WP3. Front. Microbiol. 8:1093.

doi: 10.3389/fmicb.2017.01093

\section{Genome-Wide Detection of Small Regulatory RNAs in Deep-Sea Bacterium Shewanella piezotolerans WP3}

\author{
Muhammad Z. Nawaz ${ }^{1,2}$, Huahua Jian ${ }^{1}$, Ying He ${ }^{1,2}$, Lei Xiong ${ }^{1}$, Xiang Xiao ${ }^{1,2}$ and \\ Fengping Wang ${ }^{1,2 *}$
}

${ }^{1}$ State Key Laboratory of Microbial Metabolism, School of Life Sciences and Biotechnology, Shanghai Jiao Tong University, Shanghai, China, ${ }^{2}$ State Key Laboratory of Ocean Engineering, Shanghai Jiao Tong University, Shanghai, China

Shewanella are one of the most abundant Proteobacteria in the deep-sea and are renowned for their versatile electron accepting capacities. The molecular mechanisms involved in their adaptation to diverse and extreme environments are not well understood. Small non-coding RNAs (sRNAs) are known for modulating the gene expression at transcriptional and posttranscriptional levels, subsequently playing a key role in microbial adaptation. To understand the potential roles of sRNAs in the adaptation of Shewanella toward deep-sea environments, here an in silico approach was utilized to detect the sRNAs in the genome of Shewanella piezotolerans WP3, a piezotolerant and psychrotolerant deep-sea iron reducing bacterium. After scanning 3673 sets of $5^{\prime}$ and $3^{\prime}$ UTRs of orthologous genes, 209 sRNA candidates were identified with high confidence in S. piezotolerans WP3. About 92\% (193 out of 209) of these putative sRNAs belong to the class trans-encoded RNAs, suggesting that trans-regulatory RNAs are the dominant class of sRNAs in S. piezotolerans WP3. The remaining 16 cis-regulatory RNAs were validated through quantitative polymerase chain reaction. Five cis-sRNAs were further shown to act as cold regulated sRNAs. Our study provided additional evidence at the transcriptional level to decipher the microbial adaptation mechanisms to extreme environmental conditions.

Keywords: sRNA, Shewanella piezoloterans WP3, deep-sea, adaptation, gene regulation

\section{TOOLS DESCRIPTION:}

QRNA, A tool used for the detection of the conserved RNA secondary structures, including both ncRNA genes and cis-regulatory RNA structures.

RNAz, A program for predicting structurally conserved and thermodynamically stable RNA secondary structures in multiple sequence alignments.

RNAalifold, A software used for the prediction of a consensus structure for a set of related RNAs.

sRNAPredict3/SIPHT, sRNApredict3/SIPHT are recent versions of the sRNApredict suite that are used in the efficient prediction of sRNAs, with a high level of specificity. SIPHT is a compatible web version of sRNAPredict 3 that searches approximately 1900 bacterial replicons from the NCBI database and predicts putative sRNA locations. sRNApredict3 is inclusive of sequence comparable options to look for conserved sRNAs. 
sRNAscanner, The sRNAscanner is a computational tool used to detect the intergenic small RNA specific transcriptional units (TU) in the completely sequenced bacterial genome.

NAPP (Nucleic acid phylogenetic profiling), is a clustering method that efficiently identifies non-coding RNA (ncRNA) elements in a bacterial genome.

Rfam, The Rfam database is a collection of RNA families, each represented by multiple sequence alignments, consensus secondary structures and covariance models (CMs).

RNA Infernal, Infernal ("INFERence of RNA ALignment") is a tool for searching DNA sequence databases for RNA structure and sequence similarities.

CLUSTALW, ClustalW is a general purpose DNA or protein multiple sequence alignment program for three or more sequences.

PAM, PAM (point accepted mutation) matrices are used as substitution matrices to score sequence alignments in CLUSTALW.

FastTree, is open-source software to infer approximately maximum-likelihood phylogenetic trees from alignments of nucleotide or protein sequences.

\section{INTRODUCTION}

Until the early 1990s, non-coding DNA was assumed to be non-functional and referred to as Junk DNA (Pagel and Johnstone, 1992). From the past two decades, these non-coding sequences were shown to act as modulators and regulators of gene expression in response to environmental signals (Gottesman, 2005; Vogel and Wagner, 2007; Waters and Storz, 2009), and were usually small in size (50-500 nucleotide [nt]) (Vogel and Papenfort, 2006; Gottesman and Storz, 2011) called small noncoding RNA (sRNA) and were shown to involve in a variety of biological processes, including quorum sensing, bacterial virulence, iron homeostasis, stress responses and so on (Masse and Gottesman, 2002; Lenz et al., 2004; Thomason et al., 2012). The sRNA-encoding genes are widespread in bacterial genomes (Luban and Kihara, 2007) and sRNAs can regulate the gene expression both at transcriptional and post-transcriptional level (Gottesman and Storz, 2011). The sRNA that binds to target mRNA can act either as cis or trans. Cis-encoded sRNA is typically encoded adjacent to its regulatory target on the same strand as a riboswitch or on the opposite strand to an antisense sRNA, with a perfect base-pairing region between their transcripts (Wagner et al., 2002; Brantl, 2007). On the other hand, trans-encoded sRNA is separated from the target gene, where an imperfect base-pairing often occurs between their transcripts (Gottesman, 2002, 2005; He and Hannon, 2004; Aiba, 2007). Trans-regulatory sRNAs are believed to be involved in the regulation of several biological processes including iron homeostasis and quorum sensing (Gottesman and Storz, 2011) while most of the cis-regulatory RNAs are known to maintain the appropriate copy number of the mobile elements (Wagner et al., 2002; Brantl, 2007). To date, almost all the sRNA species identified are encoded in the intergenic regions (IGRs) (Koo et al., 2011). IGRs are under lower selection pressure when compared to the rest of genomic regions, allowing more room to mutate and evolve in response to various environmental stimuli.

Since the first discovery of sRNA using electrophoresis in Escherichia coli in 1967 (Hindley, 1967), recent advances in 2D-gel electrophoresis, Northern blotting, direct labeling and RNA sequencing, DNA microarray, shotgun sequencing, co-purification, and genomic SELEX (Systematic Evolution of Ligands by Exponential enrichment) have led us to enhanced identification of sRNAs (Vogel and Sharma, 2005; Vogel et al., 2005; Huttenhofer and Vogel, 2006; Altuvia, 2007; Livny and Waldor, 2007; Liu et al., 2009). Previous sRNA identification studies have mainly been carried out on extensively studied bacteria including E. coli (Hershberg et al., 2003), Salmonella (Hebrard et al., 2012), Pseudomonas aeruginosa (Livny et al., 2006), Staphylococcus aureus (Marchais et al., 2009), Synechocystis PCC 6803 (Voss et al., 2009), Burkholderia pseudomallei (Khoo et al., 2012), B. cenocepacia J2315 (Ramos et al., 2012), Clostridium difficile (Soutourina et al., 2013), Brucella abortus 2308 (Dong et al., 2014), and Listeria monocytogenes (ToledoArana et al., 2009). E. coli has been the most studied microbe in this context, almost 80 sRNAs, including $30 \mathrm{Hfq}$ (host factor for Q2)-dependent ones, have been validated using various experimental approaches such as Northern blot and microarray (Altuvia, 2007; Waters and Storz, 2009). Hfq belongs to the large family of Sm and Sm-like proteins (a family of RNA binding proteins), that promotes the binding between sRNA and its target mRNA through conserved sequence motif (Møller et al., 2002). sRNAs such as $R y h B$ (regulate iron homeostasis) have been characterized using microarrays. OxyR (oxidative stress induced RNA), and $\operatorname{CsrB}$ (carbon storage regulator) were discovered by co-purification with overproduced CsrA protein (Altuvia et al., 1997; Romeo, 1998). Growth phase dependent sRNA genes in E. coli and S. aureus were identified using DNA microarray along with comparative genome analysis (Wassarman et al., 2001; Pichon and Felden, 2005; Silvaggi et al., 2006). The sRNAs have also shown to play regulatory roles in response to fluctuating conditions, for instance, $R y h B$ has shown to involve in regulation of tricarboxylic acid cycle under iron-limiting conditions, through modulating the fur (ferric uptake regulator) gene expression in E. coli (Modi et al., 2011; Salvail and Masse, 2012; Michaux et al., 2014).

However, experimental methods are tedious and time-consuming. Moreover, expressions of sRNAs are condition-dependent (Stubben et al., 2014), therefore, experimental verification of sRNAs are less effective and inconclusive (McHugh et al., 2014). As a result, many of the predicted sRNAs could not be verified using experimental methods (Gottesman and Storz, 2011). Alternatively, with the availability of in silico sRNA prediction algorithms, computational screening of sRNAs in a large/genomic scale becomes efficient and complementary to experimental approaches (Livny and Waldor, 2007; Khoo et al., 2012). Biocomputationally predicted sRNAs are subsequently validated through experiments (Argaman et al., 2001; Rivas and Eddy, 2001; Wassarman et al., 2001). Recently, computational tools based on different features, such as RNA secondary structures, 
thermodynamic stability, conservation of sequence and structure, transcriptional termination signals, and non-coding sequence clusters based on cross-genome conservation profiles (Vogel and Sharma, 2005; Lu et al., 2011), have greatly facilitated the efficient prediction of sRNAs in diverse bacterial species ( $\mathrm{Lu}$ et al., 2011). Some of the widely used de novo search tools (Table 1) include QRNA (Rivas and Eddy, 2001), RNAz (Washietl and Hofacker, 2004), sRNAPredict3/SIPHT (Livny and Waldor, 2007), sRNAscanner (Aziz et al., 2010), RNAalifold (Bernhart et al., 2008), and NAPP (Nucleic acid phylogenetic profiling) (Ott et al., 2012). RNAz predicts evolutionarily conserved and thermodynamically stable RNA secondary structures in multiple sequence alignments, which is not only accurate as compared to other available tools (Xu et al., 2009) but also efficient as well (Washietl et al., 2005). Knowledge-based approaches, with homologs of identified sRNAs for profiling, can be used as complementary to de novo ones. RNA Infernal (Nawrocki and Eddy, 2007), one of the knowledge-based sRNA identification tool, together with a de novo tool RNAz were used in this study.

Not all predicted sRNAs can be verified by experimental techniques due to the fact that the functioning of sRNAs is condition-dependent. Due to the difficulty in experimental verification and characterization, only a small portion of computationally identified sRNA is subjected to testing. Up to date, a few studies on sRNA prediction and characterization have been conducted at a large scale, where at least over 100 sRNAs have been identified in each study (Table 1). In a study by Livny et al. (2006), in total 2759 sRNAs were predicted in Pseudomonas aeruginosa, but only 31 were tested and 17 were validated. According to Khoo et al. (2012), over 1300 sRNAs were identified in Burkholderia pseudomallei, 15 of which were tested and 8 were validated. Different computation tools have different sensitivities in generating sRNA candidates, varying from hundreds to thousands (Table 1), integrated use of tools provide a moderate and more accurate dataset for validation. The percentage of validated sRNAs relative to the total number of tested candidates can be as high as $100 \%$ in the case for B. cenocepacia J2315 (Ramos et al., 2012) and Synechocystis PCC6803 (Voss et al., 2009), and as low as 29\% in Staphylococcus aureus (Marchais et al., 2009) (Table 1).

Shewanella are widely distributed aquatic organisms and one of the most abundant Proteobacteria in the deep sea, with the ability to grow on minimal medium and utilize a variety of compounds, such as iron, manganese, sulfite, oxygen, chromium, uranium, nitrate, fumarate, trimethylamine $N$-oxide (TMAO), and DMSO (Dimethyl sulfoxide), as terminal electron acceptors (Kato, 1999; Kato and Nogi, 2001). In addition, Shewanella has been shown to adapt to harsh and diverse environmental conditions with extreme temperature, $\mathrm{pH}$, salinity, and pressure (Hau and Gralnick, 2007). Shewanella piezotolerans WP3 (hereafter referred as WP3), a piezotolerant and psychrotolerant Gram-negative gammaproteobacteria, was isolated from west Pacific deep-sea sediment at a depth of 1914 m (Wang et al., 2004). WP3 is considered as a good candidate for studying adaptation of Shewanella to deep-ocean (Xiao et al., 2007), as it grows in the pressure range $0.1-50 \mathrm{MPa}$ with optimal growth at $20 \mathrm{MPa}$, and in the temperature range of $0-28^{\circ} \mathrm{C}$ with optimum growth temperature of $20^{\circ} \mathrm{C}$ (Xiao et al., 2007). WP3 has shown capable of adapting to a broad range of physical environmental conditions (Wang et al., 2008; Yang et al., 2013). Among the closely related species of WP3 from Shewanella genus, S. oneidensis MR-1 (referred as MR-1) is extensively studied and environmentally important species of the Shewanella genus because of its ability to use more than ten respiratory electron acceptors including nitrate, chromium, and uranium (Tiedje, 2002). S. psychrophila WP2 (referred as WP2) was isolated along with WP3 (Xiao et al., 2007), and shown to be a facultative anaerobic and psychrophilic, growing optimally at about $10-15^{\circ} \mathrm{C}$. S. violacea DSS12 (referred as DSS12) is a piezo- and psychrophilic deepsea bacterium which grows optimally at $8^{\circ} \mathrm{C}$ and pressure of $30 \mathrm{MPa}$ (Kato et al., 1995). DSS12 and S. benthica KT99 (referred as KT99), two piezophiles (Lauro et al., 2013), are the only species belonging to Shewanella genus which is found to be present at the depth of more than $2,000 \mathrm{~m}$ in the ocean (Wang et al., 2004). Details of these 26 sequenced genomes of Shewanella genus used in this study are available in Supplementary Table S1. In the present study, we used an in silico approach to detect the sRNAs in the genome of Shewanella piezotolerans WP3 and predicted sRNAs were further characterized, validated through transcriptions and quantitative polymerase chain reaction (qPCR). Discovery of novel sRNAs in WP3 and studying their conservation patterns across deep-sea bacterial lineages will lead us to elucidate gene regulation

TABLE 1 | Summary of computational discovery and validation of bacterial sRNAs.

\begin{tabular}{|c|c|c|c|c|c|c|}
\hline \multirow[t]{2}{*}{ Bacteria species } & \multirow{2}{*}{$\begin{array}{l}\text { Computational } \\
\text { Identification method }\end{array}$} & \multirow{2}{*}{$\begin{array}{l}\text { Experimental } \\
\text { verification method }\end{array}$} & \multicolumn{3}{|c|}{ Number of sRNAs } & \multirow[t]{2}{*}{ Reference } \\
\hline & & & Predicted & Tested & Verified & \\
\hline Pseudomonas aeruginosa & sRNAPredict2 & Northern blot & 2759 & 31 & 17 & Livny et al., 2006 \\
\hline Staphylococcus aureus & NAPP & Northern blot & 189 & 24 & 7 & Marchais et al., 2009 \\
\hline Synechocystis PCC6803 & $\mathrm{RNAz}$ & Northern blot & 383 & 2 & 2 & Voss et al., 2009 \\
\hline Burkholderia pseudomallei & SIPHT, sRNAScanner and RNA Infernal & RT-PCR & 1306 & 15 & 8 & Khoo et al., 2012 \\
\hline Burkholderia cenocepacia J2315 & RNAalifold \& TransTermHP & Northern blot & NA & 24 & 24 & Ramos et al., 2012 \\
\hline Brucella abortus 2308 & SIPHT and NAPP & RT-PCR & 129 & 20 & 7 & Dong et al., 2014 \\
\hline Shewanella piezotolerans WP3 & RNAz and RNA Infernal & RT-qPCR & 209 & 16 & 15 & This study \\
\hline
\end{tabular}


and molecular mechanisms of bacterial adaptation to extreme deep-sea environmental conditions.

\section{MATERIALS AND METHODS}

\section{Intergenic Region Extraction and Orthologs Prediction}

As bacterial regulatory motifs were found to be encoded in IGRs of the genome, each IGR dataset was generated by extracting sequences from both $5^{\prime}$ and $3^{\prime}$-untranslated regions (UTRs) of each gene in WP3 (described in details below), together with its orthologous IGRs in other reference genomes (Thomason and Storz, 2010). IGRs from $5^{\prime}$ and $3^{\prime}$-UTRs of the orthologous sets of genes were extracted and considered as orthologous sets of IGRs. Orthologous relationship of genes across all the reference genomes was predicted by using OrthoMCL (Li et al., 2003). Afterward, IGRs of each gene belonging to same orthologous groups (OG) in WP3 and reference genomes were retrieved as a set of orthologous IGR.

For each IGR dataset from $5^{\prime}$-end, up to $250 \mathrm{nt}$ upstream (depending upon the length of IGR) and $20 \mathrm{nt}$ downstream of the translation initiation sites of the corresponding genes in the positive direction were extracted and considered as one orthologous set of $5^{\prime}$-UTRs and subject to RNA secondary structure prediction. For the purpose of increased specificity and sensitivity, three overlapping windows, that is $-250 \sim-100$, $-200 \sim-50$ and $-150 \sim+20$ ( 1 corresponds to the translation start site, "-" referred to upstream, and "+" to downstream) were generated from each original IGR dataset and analyzed separately. For genes transcribing in the negative direction, $250 \mathrm{nt}$ downstream and $20 \mathrm{nt}$ upstream of translation initiation sites were extracted and divided into three overlapping windows in the same manner. Similarly, for the extraction of orthologous IGR datasets from $3^{\prime}$-end, up to $250 \mathrm{nt}$ (depending upon the length of IGR) sequences downstream and $20 \mathrm{nt}$ upstream of the translation initiation sites of genes in the positive direction were retrieved as one orthologous set and subject to RNA secondary structure prediction. This IGR region was divided into three overlapping windows that are $-20 \sim 150,50-200,100-250$. For genes transcribing in the negative direction, $250 \mathrm{nt}$ upstream and $20 \mathrm{nt}$ downstream of translation initiation site were extracted and divided into three overlapping windows.

\section{sRNA Prediction by RNAz and RNA Infernal}

We applied a comparative genomics-based approach for prediction of bacterial sRNA by integrating the RNA secondary structure prediction tool RNAz (Washietl et al., 2005) and RNA motif searching tool RNA-Infernal ${ }^{1}$. RNAz is widely used and comparatively reliable for de novo detection of structured non-coding RNAs from comparative genomics analysis (Washietl et al., 2005; Gruber et al., 2010). This program predicts structurally conserved and thermodynamically stable RNA

${ }^{1}$ http://eddylab.org/infernal/ secondary structures from multiple sequence alignments, with training data from multiple Rfam families and RNAalifold (Bernhart et al., 2008) for common RNA structure prediction. RNA Infernal is a homology-based tool that searches sequence databases for homologs of structural RNA sequences and then generates structure-based RNA sequence alignments. As stated above, IGRs were extracted from $5^{\prime}$ and $3^{\prime}$-UTRs of genes in target genome and its orthologous regions in reference genomes. Afterward, multiple sequence alignment for each IGR orthologous dataset was performed with CLUSTALW (Thompson et al., 1994) (using PAM substitution matrix). RNAz was then applied on multiple aligned sequences, with default parameters. Orthologous UTR sets that were predicted as RNA by RNAz were searched in Rfam database using the RNA motif searching software Infernal (Nawrocki and Eddy, 2007), where highly confident candidates were those, which predicted with an infernal score higher than 10 bit (CM score $>10)$.

\section{Conservation of Predicted sRNAs}

We used the same approach to find the conservation or genome specificity of predicted cis-sRNAs in few closely related species of Shewanella genus and well-studied piezophilic and psychrophilic bacteria. We retrieved the $5^{\prime}$-UTR of a gene in WP3 and $5^{\prime}$-UTRs of orthologs of this gene in other species of Shewanella genus or other genera as a set of orthologous IGR. We applied RNAz on these orthologous sets of IGRs, positively predicted sets by RNAz were further subject to RNA Infernal. If the sequence in the set of orthologous IGR that belongs to $5^{\prime}$-UTR in WP3 and have infernal score higher than 10, it was declared as sRNA in WP3. Other sequences belonging to other species in the same set of orthologous IGR were considered as conserved if they also gave an infernal score higher than 10. These predicted sRNAs are conserved sRNAs because they are orthologous to each other as they were found at the $5^{\prime}$-UTR of the orthologous genes.

\section{Phylogenetic Tree Construction}

Maximum-likelihood tree based on 16S rRNA gene sequences was constructed to represent the taxonomic relationships of the species used in this study and tree was used to demonstrate the conservation pattern of 16 identified cissRNAs across species used in present study. 16S rRNA gene sequences of all the representative species were aligned using CLUSTALW and tree was constructed with FastTree using default parameters. Bootstrap values are based on 1000 replicates and are shown with white $(\geq 80 \%)$ and black $(\geq 90 \%)$ circles (Figure 1).

\section{Culture Conditions and RNA Isolation}

Shewanella piezotolerans WP3 was cultured in a modified marine medium $2216 \mathrm{E}$ (5 g/liter tryptone, $1 \mathrm{~g} /$ liter yeast extract, $0.1 \mathrm{~g} /$ liter $\mathrm{FePO}_{4}, 34 \mathrm{~g} /$ liter $\mathrm{NaCl}$ ) (Xiao et al., 2007). For aerobic cultivation, the single clone of WP3 strains were firstly inoculated into a $5 \mathrm{ml}$ test tube, then the culture was diluted 1000 fold in the same medium with shaking $(220 \mathrm{rpm})$ at $0.1 \mathrm{MPa}$ (1 atm) and 20 and $4^{\circ} \mathrm{C}$, respectively. For anaerobic cultivation, 


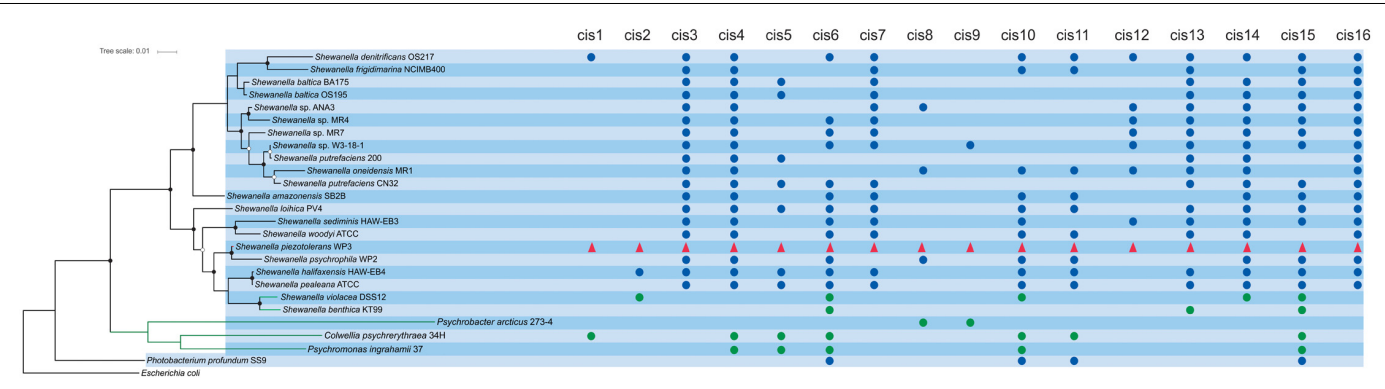

FIGURE 1 | Phylogenetic relationships of the species used in this studies and conservation pattern of 16 identified sRNAs. 165 rRNA gene sequences based maximum-likelihood tree of species used in this studies shows their taxonomic relationship. Bootstrap values are based on 1000 replicates and are shown with white $(\geq 80 \%)$ and black $(\geq 90 \%)$ circles. sRNAs in WP3 are shown in red triangles, sRNAs in psychrophilic species are shown in green circles while sRNAs in other related species are indicated with blue circles.

media was prepared without any electron acceptor under nonsterile condition. Prepared media was dispensed into serum bottles filled with $\mathrm{O}_{2}$-free nitrogen, and bottles were covered with stoppers. Metal seals were used to seal the caps and media was autoclaved by inserting a needle into the stopper. Needles were plucked off immediately after the autoclave was done. Then serum bottles were filled by a gassing manifold system after the media was cooled down (Balch and Wolfe, 1976). Nitrate solution (0.4 M, sodium nitrate) was filter sterilized in a vinyl anaerobic airlock chamber (Coy Laboratory Products Inc., Grass Lake, MI, United States) and added to the concentration needed. The serum bottles, stoppers, and metal seals were bought from Wheaton Science Products (Millville, NJ, United States). In order to incubate WP3 at high pressure, log phase cultures of WP3 cells at atmospheric pressure were grown and then diluted 1000-fold with the same medium for anaerobic cultivation. After that cells were transferred into sterile injection syringes and were placed inside the pressure vessels. The syringes were then incubated at a hydrostatic pressure of $20 \mathrm{MPa}(200 \mathrm{~atm})$ at $4^{\circ} \mathrm{C}$. Pin closure pressure vessels were used in this study (Feiyu Petrochemical Instrument Equipment Inc., Nantong, China). Pressure was applied using a hand-operated pump with a quick-fit connector to the pressure vessel.

The growth of the WP3 strains was determined using turbidity measurements at $600 \mathrm{~nm}$ with a spectrophotometer (UV-2550, Shimadzu, Kyoto, Japan). The culture of WP3 was collected immediately when the cells reached mid-exponential phase $\left(\mathrm{OD}_{600} \approx 0.8\right.$ and 0.2 for aerobic and anaerobic cultivation, respectively). The samples were centrifuged for $30 \mathrm{~s}$ at the maxim speed $(16000 \times g)$. The cells were immediately frozen in liquid nitrogen for subsequent RNA extraction.

TRI reagent-RNA/DNA/protein isolation kit (MRC, Cincinnati, OH, United States) was used to isolate the total RNA. After treating the RNA samples with DNase I at $37^{\circ} \mathrm{C}$ for $1 \mathrm{~h}$, RNA was purified by using RNeasy Mini Kit (Qiagen, Hilden, Germany). The quality of RNA samples were determined by visualizing the nearly $2: 1$ ratio of $23 \mathrm{~S}: 16 \mathrm{~S}$ ribosomal RNA by running a $1.0 \%$ TAE (Tris-Acitate-EDTA) agarose gel. The total RNA was treated with DNase I at $37^{\circ} \mathrm{C}$ for $1 \mathrm{~h}$ to remove DNA contamination and the purity was checked by PCR amplification with RNA as template. The quantity and integrity of RNA was evaluated with a UV spectrophotometer (Thermo Fisher, Waltham, MA, United States). In general, the ratio of $260 \mathrm{~nm} / 280 \mathrm{~nm}>2$ and $260 \mathrm{~nm} / 280 \mathrm{~nm} \approx 2.2$ indicate the RNA is pure and could be used for the follow-up analysis. cDNA was synthesized from the purified RNA samples with the RevertAid First Strand cDNA Synthesis Kit (Fermentas, Glen Burnie, MD, United States).

\section{Microarray Analysis of Differentially Expressed Genes at Low Temperature}

The expression profiles of genes in WP3 genome at low temperature $\left(4^{\circ} \mathrm{C}\right.$ compared to $\left.25^{\circ} \mathrm{C}\right)$ were studied using microarray data (NCBI GEO dataset accession: GSE80668). A microarray that contained $95 \%$ of the total predicted genes of WP3 was designed and manufactured (CapitalBio, Beijing, China). Differentially expressed genes (DEGs) were the ones with a significant change (fold $\geq 2$ ) in expression pattern. Microarray signals with $P$-values $<0.001$ in the $F$-test were regarded as DEGs. All of the DEGs were confirmed with the Significance Analysis of Microarrays (SAM) software. Detailed descriptions of the microarray procedure for WP3 under cold temperature was described somewhere else (Jian et al., 2016).

\section{Real-Time qPCR}

Primer Express software (ABI) was used to design the primer pairs for the selected genes for real-time $\mathrm{qPCR}$ (qPCR). PCR cycling was conducted using 7500 System SDS software (ABI, Foster City, CA, United States) in reaction mixtures with total volumes of $20 \mu \mathrm{l}$ containing $1 \times$ SYBR Green I Universal PCR Master Mix (ABI, Foster City, CA, United States), $0.5 \mu \mathrm{M}$ each primer, $1 \mu \mathrm{l}$ cDNA template. The amount of target was normalized to that of the reference gene swp2079, whose expression remains constant under various conditions relative to the calibrator (The transcription levels of the genes at $20^{\circ} \mathrm{C}$ and $0.1 \mathrm{MPa}$ were set as 1) (Li et al., 2006). qPCR assays were performed in triplicate for each sample, and a mean value and standard deviation were calculated for the relative RNA expression 
levels. Primers designed for qPCR are shown in Supplementary Table S2.

\section{RESULTS}

sRNAs are considered to be evolutionarily conserved in their secondary structures among the closely related species (Livny and Waldor, 2007). Here, we used a comparative genomicsbased approach for genome-wide screening of sRNAs. At present, various species of Shewanella genus from various environments have been sequenced. In this comparative genomics-based approach, 26 representatives sequenced genomes of Shewanella genus were selected based on niche and phylogenetic relatedness and being well-studied and were used as reference genomes, including one partial sequenced genome (Shewanella benthica KT99), being closely related to WP3. We have also included a genome sequence of Shewanella psychrophila WP2 isolated previously along with WP3 (Wang et al., 2004). This unpublished completely sequenced genome was also studied and discussed here in this study. In addition, we also investigated the distribution pattern of the identified cis-regulatory RNAs in WP3, across 10 bacterial species for piezophilic and psychrophilic adaptation features, including four selected Shewanella species (MR-1, WP2, DSS12, and KT99), three piezophilic model bacteria [Photobacterium profundum SS9 (Vezzi et al., 2005), Marinitoga piezophila KA3 (Lucas et al., 2012), Desulfovibrio piezophilus C1TLV30 (Khelaifia et al., 2011), referred as SS9, KA3, and C1TLV30], and three well studied psychrophilic bacteria (Colwellia psychrerythraea $34 \mathrm{H}$, Psychromonas ingrahamii 37, Psychrobacter arcticus 273-4, referred as $\mathrm{Cp} 34 \mathrm{H}, \mathrm{Pi} 37$, and $\mathrm{Pa} 273-4)$.

\section{sRNA Prediction in WP3 and Other Shewanella Species}

Using an integrated approach 209 RNA motifs in WP3 were predicted as reliable sRNA candidates. For comparative analysis of occurrence of sRNAs and determining the conservation of sRNAs, genomes of closely related species including MR-1, WP2, DSS12, and KT99 were scanned for sRNAs identification, using the same approach (Table 2). From total sets of $5^{\prime}$ and $3^{\prime}$ UTRs in WP3 and other four Shewanella species, about 3 to $8 \%$ were predicted as sRNA candidates. As cis-regulatory RNAs are often located in the $5^{\prime}$-UTR of the mRNA (Xu et al., 2009), predicted sRNAs from $5^{\prime}$-UTRs are considered as cisregulatory and sRNAs from $3^{\prime}$-UTRs as trans-regulatory RNAs (Papenfort et al., 2015). Cis-regulatory RNAs are mainly known to maintain the appropriate copy number of the mobile elements (Wagner et al., 2002; Brantl, 2007), while trans-regulatory RNAs are associated with almost every global response in bacteria (Gottesman and Storz, 2011). Because of their ubiquitous roles, trans-acting sRNAs are extensively studied and well characterized in Gram-negative bacteria (Man et al., 2011). However, compared to trans-sRNAs, cis-sRNAs are much less understood. In the present study, the proportion of predicted cis-encoded sRNA was lower than that of trans-encoded sRNAs in WP3, with a ratio for

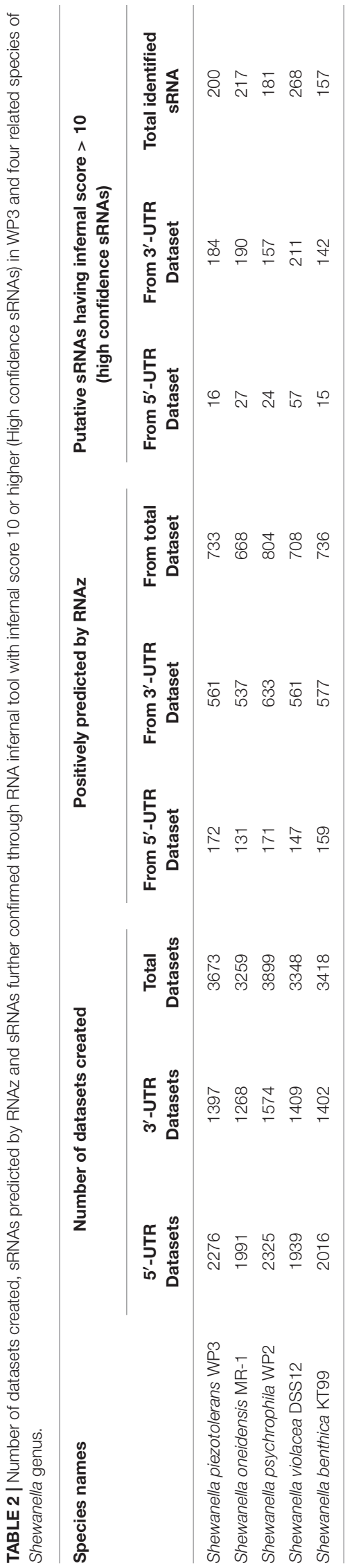

Frontiers in Microbiology | www.frontiersin.org 
cis- and trans-sRNA 1:12, and the roles of all the 16 predicted cissRNA (referred as cis1-16 herein) in WP3 will be discussed here in this paper.

\section{Conservation of Identified Cis-sRNA}

The distribution pattern of the 16 identified cis-regulatory RNAs in WP3 across selected piezophilic and psychrophilic bacterial species (details in Materials and Methods) was displayed in Figure 1. Seven sRNAs (cis2, cis3, cis7, cis12, cis13, cis14, and cis16) were found limited to Shewanella genus, so they are more likely to be Shewanella specific sRNAs. Nine out of the 16 predicted cis-regulatory RNAs have been found universally conserved in at least half of all the reference genomes presented in this study (Figure 1). Only one piezophilic species Photobacterium profundum SS9 shared four conserved cis-encoded sRNA with WP3, while in the rest of two piezophiles (Marinitoga piezophila KA3 and Desulfovibrio piezophilus C1TLV30) no common sRNAs was identified. One of these four conserved cis-sRNAs in Photobacterium profundum SS9 (conserve with cis15 in WP3) was already identified in a study by Campanaro et al. (2012) (locus tag = PBPRA0551b). All the four psychrophilic species used in the present study shared conserved cis-sRNAs with WP3, where five sRNA (cis4, cis5, cis6, cis10, and cis15) were conserved in at least half of the all psychrophilic species used in this study (Figure 1).

\section{Function Inference from Genome Annotation}

All 16 cis-regulatory RNAs were annotated against the known regulatory motifs in Rfam database (Table 3). Among the five cis-sRNAs conserved in psychrophilic bacteria, cis4 was annotated as asX2, an sRNA which appears to function in virulence (Schmidtke et al., 2012). cis5 was annotated as rimP with a possible role in the regulation of NusA protein (a protein which functions in $30 \mathrm{~S}$ ribosomal subunit maturation) (Nord et al., 2009; Naville and Gautheret, 2010), cis6 as $n s i R$ with a function in cell differentiation (Muro-Pastor, 2014). cis10 was with the best hit as Atu_C9, with reported roles in growth (Wilms et al., 2012), while cis15 was with the best hit to STnc180 with unknown function (Sittka et al., 2008). According to the genome annotation of WP3, the $5^{\prime}$ genes of both cis 4 and cis5 is anthranilate synthase component I (TrpE), an enzyme involved in the conversion of chorismate to anthranilate, while the $5^{\prime}$ gene of cis6 is 2-isopropylmalate synthase, which plays an important role in the biosynthesis of l-leucine and pyruvate metabolism (Webster and Gross, 1965; Cole et al., 1973). The 5' gene for cis10 is ATP phosphoribosyltransferase (biosynthesis of histidine), and for cis15 is aspartate kinase (phosphorylation of the amino acid aspartate). Locations of identified cis-sRNAs and their neighborhood genes in the genome of WP3 are shown in Supplementary Figure S1.

\section{Validation Using Microarray Data and qPCR}

As cis-regulatory RNAs are responsible for regulating the gene expression of their $5^{\prime}$-associated genes, gene expression of identified cis-sRNAs and their associated genes at optimum and cold temperature conditions were determined using RT-qPCR and microarray data, respectively. Five cis-sRNA (cis4, cis5, cis6, cis10, and cis15) were appeared conserve in psychrophilic species and were considered to have a role in cold adaptation and later qPCR analysis showed their increased expression under all cold conditions (Figure 2), therefore regarded as cold-regulated sRNAs. Interestingly, $5^{\prime}$-genes of all the five cold-regulated sRNAs were shown to be up-regulated under cold temperature conditions (Table 3). As cis-regulatory RNAs in bacteria are typically located at the $5^{\prime}$-end of their target genes and are involved in regulating the gene expression of their $5^{\prime}$-associated gene (Weinberg et al., 2010). Therefore, it is inferred that these predicted cis-sRNAs present at the $5^{\prime}$-end of the gene are contributing toward regulating the expression of their $5^{\prime}$-associated gene. All the 16 cis-sRNAs were also validated through RT-qPCR except cis5, a possible explanation of this exception can be that it might be expressed under some other specific conditions. qPCR results showed that 9 cis-sRNAs (cis4, cis6-11, cis14-15), including four cold regulators were present with an increase in transcription under all three low-temperature conditions (Figure 2). As these cold-regulated sRNAs were found with increased transcription under cold temperature conditions consistent with their target regulatory genes, it shows that they have positive regulatory effect over their $5^{\prime}$-associated genes, i.e., in response to cold temperature condition, increase in the sRNA transcription takes place which promotes the transcription (up-regulation) of their target regulatory genes.

\section{DISCUSSION}

In present study, we extracted the IGR both from $5^{\prime}$ and $3^{\prime}$-UTR of the orthologous genes in WP3 and reference genomes and applied combination of RNAz and RNA Infernal tool to predict sRNAs in our target bacteria WP3 and four related species of Shewanella genus, i.e., S. oneidensis MR-1, S. psychrophila WP2, S. violacea DSS12, and S. benthica KT99. Positively predicted sRNAs based on thermodynamic stability by RNAz were subjected to motif searching tool RNA infernal. sRNA candidates present at the $5^{\prime}$-UTRs which were positively predicted by RNAz and giving an infernal score higher than 10 bit were called as "confident candidates" (Xu et al., 2009), and were subsequently selected for verification through RT-qPCR. In addition to CM bit Score (Covariance model), trusted cutoffs (TC) bit scores thresholds were also used in the model to evaluate sRNAs candidates. As TC thresholds are generally considered to be the score of lowest-scoring known true positive, about half of putative cis-sRNAs validated through RT-qPCR were missed, when TC bit score thresholds (-cut_tc parameter for using CM's trusted cutoff thresholds) was used. In this study, we mainly focused on the less understood type of sRNAs (cissRNA) and reported that cis-regulatory RNAs might have a role in adaptation to extreme conditions. We also explored the conservation of cis-sRNAs in other reference species and their conservation pattern demonstrates that most of the sRNAs 


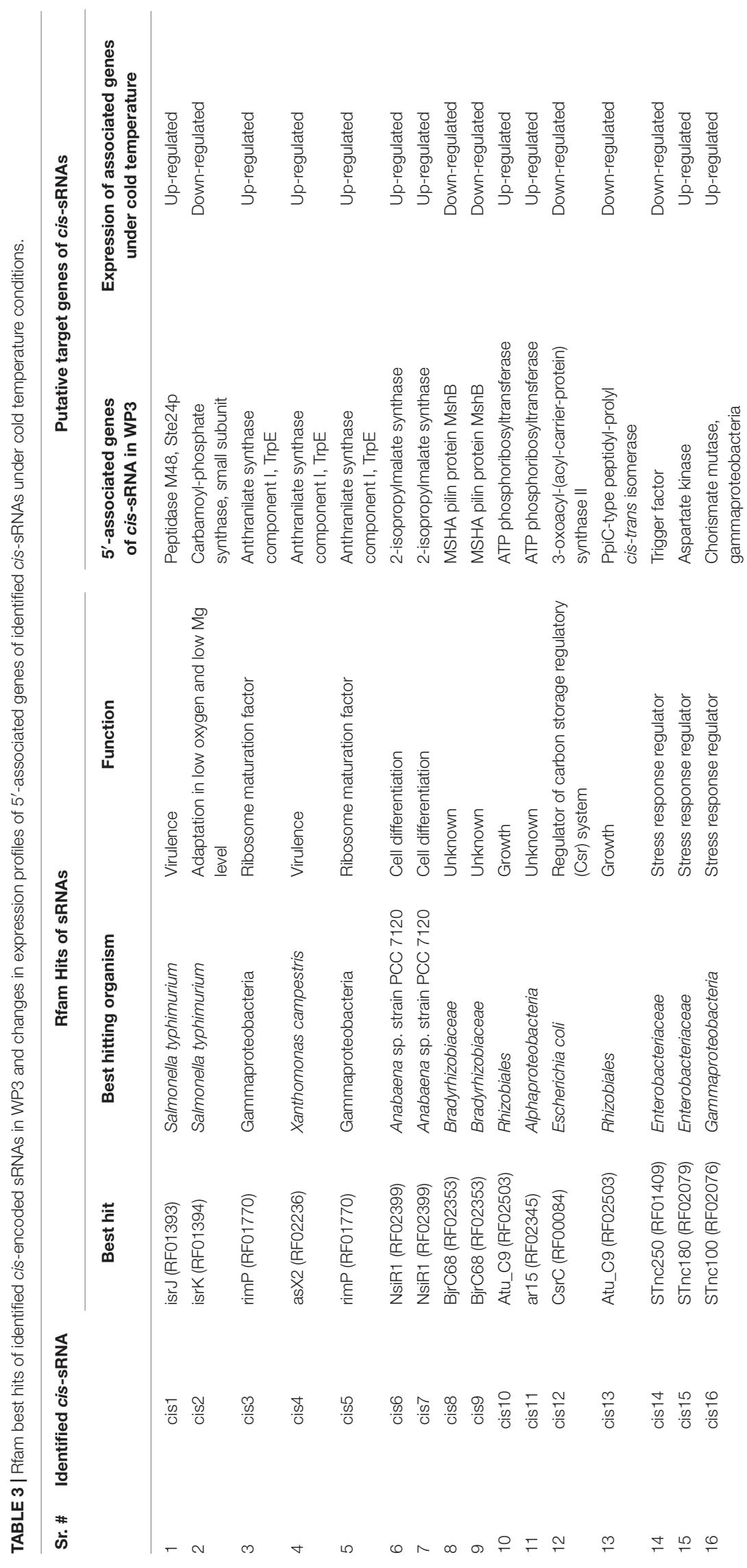




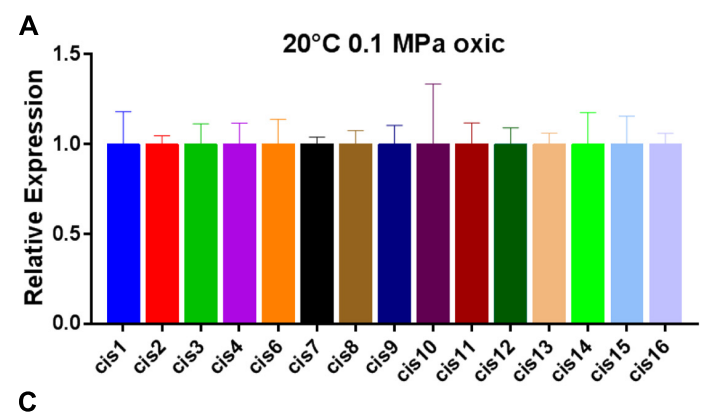

C

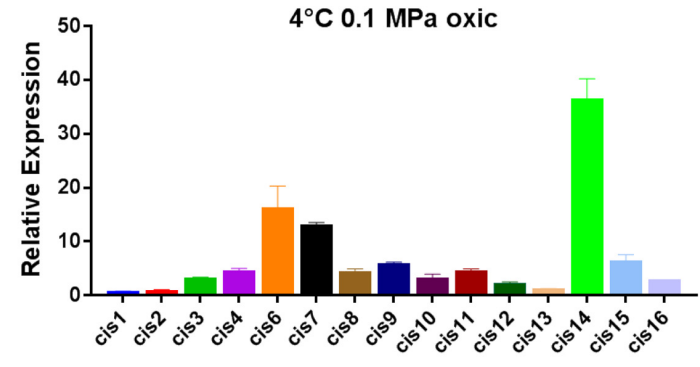

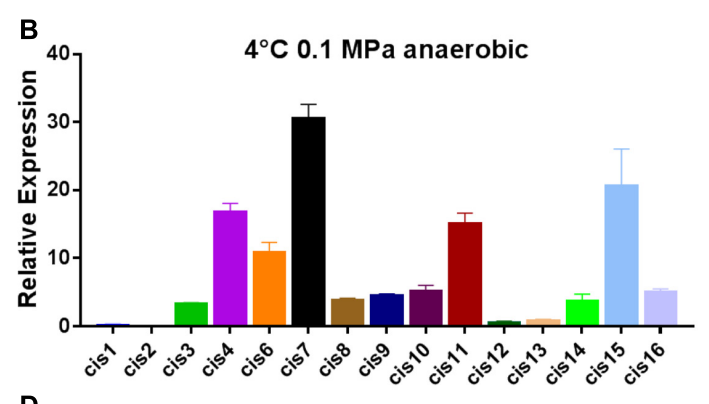

D

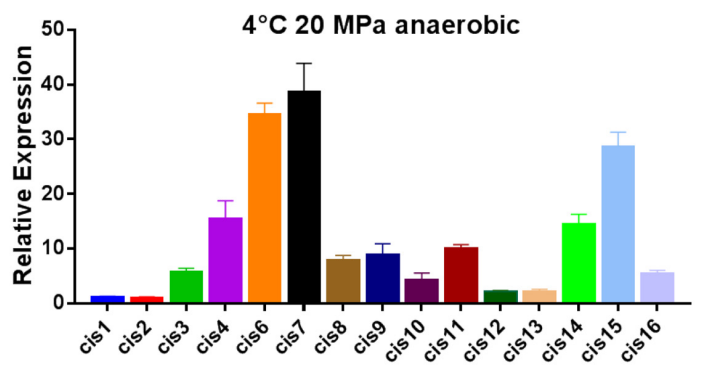

FIGURE 2 | Expression of identified cis-sRNAs under different conditions determined by qPCR. (A) Relative expression of identified cis-sRNAs at $20^{\circ} \mathrm{C}, 0.1 \mathrm{MPa}$, and under oxic conditions. (B) Relative expression of identified cis-sRNAs at $4^{\circ} \mathrm{C}, 0.1 \mathrm{MPa}$, and under oxic conditions. (C) Relative expression of identified cis-sRNAs at $4^{\circ} \mathrm{C}, 0.1 \mathrm{MPa}$, and under anaerobic conditions. (D) Relative expression of identified cis-sRNAs at $4^{\circ} \mathrm{C}, 20 \mathrm{MPa}$, and under anaerobic conditions.

tend to remain to conserve within the genus. Conservation pattern of cis-sRNAs showed that five of them were conserved not only in psychrophilic species from Shewanella genus but also in species belonging to other genera. Furthermore, we compared the expression of their $5^{\prime}$-associated genes at an optimum and cold temperature and surprisingly, expression of all the $5^{\prime}$-associated genes were found with an increase in expression at low temperature. Regulated genes of all the five conserved sRNAs in psychrophiles are involved in the biosynthesis and metabolism of amino acids, secondary metabolites, and antibiotics, suggesting that bacterium tends to increase the metabolism of amino acids, secondary metabolites, and antibiotics at cold temperature.

As most studied class of sRNAs, trans-regulatory RNAs were found to be associated with almost every global response in bacteria and were reported as an abundantly existing class of regulatory RNAs in bacterial genomes (Gottesman and Storz, 2011). Likewise, about 92\% (193 out of 209) of the sRNAs identified in this study, belonging to the class trans-encoded RNAs, which reflects that trans-regulatory RNAs are the dominant class of regulatory RNAs in S. piezotolerans WP3. We screened the orthologs of trans-encoded sRNAs in Rfam database where most of the trans-sRNAs shared homology with known trans-regulatory RNAs in E. coli, S. enterica, Staphylococcus aureus, Cyanobacterium, Xanthomonas campestris, Vibrio cholera, Listeria monocytogenes, and Rhizobiales species performing a variety of biological roles. Detailed information about the trans-sRNAs in the genome of $S$. piezotolerans WP3 and their orthologs in Rfam are available in Supplementary Table S3.
In short, we identified sRNAs in a piezo- and psychrotolerant, iron reducing, deep-sea bacterium, Shewanella piezotolerans WP3 and four closely related species of WP3. In total, we identified 209, 217, 181, 268, and 157 sRNAs in WP3, MR-1, WP2, DSS12, and KT99, respectively. Out of 209 predicted sRNAs in WP3, 16 are the cis-sRNAs (cis1-16) and were further characterized, validated through qPCR. Seven of this 16 cissRNAs (cis2, cis3, cis7, cis12, cis13, cis14, and cis16) were found as Shewanella-specific while rest of nine cis-regulatory RNAs were shown to be universal in at least half of all the genomes presented in this study. Five cis-sRNA (cis4, cis5, cis6, cis10, and cis15) were found as regulators commonly present in most of psychrophilic species used in this study. Expression analysis of 16 cis-sRNAs and their target genes demonstrated significant change under low-temperature conditions. Although the present study does not provide insights into the functioning of all the identified sRNAs (including cis and trans) in Shewanella piezotolerans WP3, further analysis is required to reveal the possible roles of these sRNAs in WP3. However, our study also provides evidence that not only trans- but cis-sRNAs could also play their roles in adaptation to extreme conditions. It should be very useful to explore the conserved sRNAs in extremophiles adapted to diverse environmental conditions.

\section{AUTHOR CONTRIBUTIONS}

FW, XX, YH, and MN designed the experiment and analysis. MN designed the pipeline and performed the computational analysis. LX conducted experiments. MN, HJ, YH and FW wrote the manuscript, in consultation with all other authors. 


\section{ACKNOWLEDGMENTS}

We are thankful to National Natural Science Foundation of China (Grant No. 31290232), China Ocean Mineral Resources R\&D Association (Grant No. DY125-22-04), and National Natural Science Foundation of China (Grant No. 41576129, 91228201, 91428308) for supporting our research. Moreover Higher Education Commission (HEC), Pakistan is gratefully acknowledged for providing financial support to $\mathrm{MN}$.

\section{REFERENCES}

Aiba, H. (2007). Mechanism of RNA silencing by Hfq-binding small RNAs. Curr. Opin. Microbiol. 10, 134-139. doi: 10.1016/j.mib.2007.03.010

Altuvia, S. (2007). Identification of bacterial small non-coding RNAs: experimental approaches. Curr. Opin. Microbiol. 10, 257-261. doi: 10.1016/j.mib.2007. 05.003

Altuvia, S., Weinstein-Fischer, D., Zhang, A., Postow, L., and Storz, G. (1997). A small, stable RNA induced by oxidative stress: role as a pleiotropic regulator and antimutator. Cell 90, 43-53. doi: 10.1016/s0092-8674(00)80312-8

Argaman, L., Hershberg, R., Vogel, J., Bejerano, G., Wagner, E. G. H., Margalit, H., et al. (2001). Novel small RNA-encoding genes in the intergenic regions of Escherichia coli. Curr. Biol. 11, 941-950. doi: 10.1016/s0960-9822(01)00270-6

Aziz, R. K., Sridhar, J., Narmada, S. R., Sabarinathan, R., Ou, H.-Y., Deng, Z., et al. (2010). sRNAscanner: a computational tool for intergenic small RNA detection in bacterial genomes. PLoS ONE 5:e11970. doi: 10.1371/journal.pone.0011970

Balch, W. E., and Wolfe, R. (1976). New approach to the cultivation of methanogenic bacteria: 2-mercaptoethanesulfonic acid (HS-CoM)-dependent growth of Methanobacterium ruminantium in a pressurized atmosphere. Appl. Environ. Microbiol. 32, 781-791.

Bernhart, S. H., Hofacker, I. L., Will, S., Gruber, A. R., and Stadler, P. F. (2008). RNAalifold: improved consensus structure prediction for RNA alignments. BMC Bioinformatics 9:474. doi: 10.1186/1471-2105-9-474

Brantl, S. (2007). Regulatory mechanisms employed by cis-encoded antisense RNAs. Curr. Opin. Microbiol. 10, 102-109. doi: 10.1016/j.mib.2007.03.012

Campanaro, S., De Pascale, F., Telatin, A., Schiavon, R., Bartlett, D. H., and Valle, G. (2012). The transcriptional landscape of the deep-sea bacterium Photobacterium profundum in both a toxR mutant and its parental strain. $B M C$ Genomics 13:567. doi: 10.1186/1471-2164-13-567

Cole, F. E., Kalyanpur, M. G., and Stevens, C. M. (1973). Absolute configuration of $\alpha$-isopropylmalate and the mechanism of its conversion to $\beta$-isopropylmalate in the biosynthesis of leucine. Biochemistry 12, 3346-3350. doi: 10.1021/ bi00741a031

Dong, H., Peng, X., Wang, N., and Wu, Q. (2014). Identification of novel sRNAs in Brucella abortus 2308. FEMS Microbiol. Lett. 354, 119-125. doi: 10.1111/15746968.12433

Gottesman, S. (2002). Stealth regulation: biological circuits with small RNA switches. Genes Dev. 16, 2829-2842. doi: 10.1101/gad.1030302

Gottesman, S. (2005). Micros for microbes: non-coding regulatory RNAs in bacteria. Trends Genet. 21, 399-404. doi: 10.1016/j.tig.2005.05.008

Gottesman, S., and Storz, G. (2011). Bacterial small RNA regulators: versatile roles and rapidly evolving variations. Cold Spring Harb. Perspect. Biol. 3:a003798. doi: $10.1101 /$ cshperspect.a003798

Gruber, A. R., Findeiss, S., Washietl, S., Hofacker, I. L., and Stadler, P. F. (2010). RNAz 2.0: improved noncoding RNA detection. Pac. Symp. Biocomput. 15, 69-79.

Hau, H. H., and Gralnick, J. A. (2007). Ecology and biotechnology of the genus Shewanella. Annu. Rev. Microbiol. 61, 237-258. doi: 10.1146/annurev.micro.61. 080706.093257

He, L., and Hannon, G. J. (2004). MicroRNAs: small RNAs with a big role in gene regulation. Nat. Rev. Genet. 5, 522-531. doi: 10.1038/nrg1379

Hebrard, M., Kroger, C., Srikumar, S., Colgan, A., Handler, K., and Hinton, J. C. (2012). sRNAs and the virulence of Salmonella enterica serovar Typhimurium. RNA Biol. 9, 437-445. doi: 10.4161/rna.20480

\section{SUPPLEMENTARY MATERIAL}

The Supplementary Material for this article can be found online at: http://journal.frontiersin.org/article/10.3389/fmicb. 2017.01093/full\#supplementary-material

FIGURE S1 | Identified cis-sRNAs in WP3 genomes and their flanking genes. Neighborhood genes are shown in green wedges and sRNAs are shown in blue colored round corner rectangles. Genes without any labels are the hypothetical proteins.

Hershberg, R., Altuvia, S., and Margalit, H. (2003). A survey of small RNAencoding genes in Escherichia coli. Nucleic Acids Res. 31, 1813-1820. doi: $10.1093 / \mathrm{nar} / \mathrm{gkg} 297$

Hindley, J. (1967). Fractionation of 32P-labelled ribonucleic acids on polyacrylamide gels and their characterization by fingerprinting. J. Mol. Biol. 30, 125-136. doi: 10.1016/0022-2836(67)90248-3

Huttenhofer, A., and Vogel, J. (2006). Experimental approaches to identify noncoding RNAs. Nucleic Acids Res. 34, 635-646. doi: 10.1093/nar/gkj469

Jian, H., Li, S., Tang, X., and Xiao, X. (2016). A transcriptome resource for the deepsea bacterium Shewanella piezotolerans WP3 under cold and high hydrostatic pressure shock stress. Mar. Genomics 30, 87-91. doi: 10.1016/j.margen.2016. 09.004

Kato, C. (1999). In Extremophiles in Deep- Sea Environments. Tokyo: Springer.

Kato, C., and Nogi, Y. (2001). Correlation between phylogenetic structure and function: examples from deep-sea Shewanella. FEMS Microbiology Ecology 35, 223-230. doi: 10.1111/j.1574-6941.2001.tb00807.x

Kato, C., Sato, T., and Horikoshi, K. (1995). Isolation and properties of barophilic and barotolerant bacteria from deep-sea mud samples. Biodivers. Conserv. 4, 1-9. doi: 10.1007/bf00115311

Khelaifia, S., Fardeau, M.-L., Pradel, N., Aussignargues, C., Garel, M., Tamburini, C., et al. (2011). Desulfovibrio piezophilus sp. nov., a piezophilic, sulfate-reducing bacterium isolated from wood falls in the Mediterranean Sea. Int. J. Syst. Evolut. Microbiol. 61, 2706-2711. doi: 10.1099/ijs.0.028670-0

Khoo, J. S., Chai, S. F., Mohamed, R., Nathan, S., and Firdaus-Raih, M. (2012). Computational discovery and RT-PCR validation of novel Burkholderia conserved and Burkholderia pseudomallei unique sRNAs. BMC Genomics 13(Suppl. 7):S13. doi: 10.1186/1471-2164-13-S7-S13

Koo, J. T., Alleyne, T. M., Schiano, C. A., Jafari, N., and Lathem, W. W. (2011). Global discovery of small RNAs in Yersinia pseudotuberculosis identifies Yersinia-specific small, noncoding RNAs required for virulence. Proc. Natl. Acad. Sci. U.S.A. 108, E709-E717. doi: 10.1073/pnas.1101655108

Lauro, F. M., Chastain, R. A., Ferriera, S., Johnson, J., Yayanos, A. A., and Bartlett, D. H. (2013). Draft genome sequence of the deep-sea Bacterium Shewanella benthica Strain KT99. Genome Announc. 1:e00210-13. doi: 10.1128/genomeA. 00210- 13

Lenz, D. H., Mok, K. C., Lilley, B. N., Kulkarni, R. V., Wingreen, N. S., and Bassler, B. L. (2004). The small RNA chaperone Hfq and multiple small RNAs control quorum sensing in Vibrio harveyi and Vibrio cholerae. Cell 118, 69-82. doi: 10.1016/j.cell.2004.06.009

Li, L., Stoeckert, C. J. Jr., and Roos, D. S. (2003). OrthoMCL: identification of ortholog groups for eukaryotic genomes. Genome Res. 13, 2178-2189. doi: 10.1101/gr.1224503

Li, S., Xiao, X., Li, J., Luo, J., and Wang, F. (2006). Identification of genes regulated by changing salinity in the deep-sea bacterium Shewanella sp. WP3 using RNA arbitrarily primed PCR. Extremophiles 10, 97-104. doi: 10.1007/s00792-0050476-x

Liu, J. M., Livny, J., Lawrence, M. S., Kimball, M. D., Waldor, M. K., and Camilli, A. (2009). Experimental discovery of sRNAs in Vibrio cholerae by direct cloning, 5S/tRNA depletion and parallel sequencing. Nucleic Acids Res. 37:e46. doi: 10.1093/nar/gkp080

Livny, J., Brencic, A., Lory, S., and Waldor, M. K. (2006). Identification of 17 Pseudomonas aeruginosa sRNAs and prediction of sRNA-encoding genes in 10 diverse pathogens using the bioinformatic tool sRNAPredict2. Nucleic Acids Res. 34, 3484-3493. doi: 10.1093/nar/gkl453 
Livny, J., and Waldor, M. K. (2007). Identification of small RNAs in diverse bacterial species. Curr. Opin. Microbiol. 10, 96-101. doi: 10.1016/j.mib.2007.03. 005

Lu, X., Goodrich-Blair, H., and Tjaden, B. (2011). Assessing computational tools for the discovery of small RNA genes in bacteria. RNA 17, 1635-1647. doi: 10.1261/rna.2689811

Luban, S., and Kihara, D. (2007). Comparative genomics of small RNAs in bacterial genomes. OMICS 11, 58-73. doi: 10.1089/omi.2006.0005

Lucas, S., Han, J., Lapidus, A., Cheng, J.-F., Goodwin, L. A., Pitluck, S., et al. (2012). Complete genome sequence of the thermophilic, piezophilic, heterotrophic bacterium Marinitoga piezophila KA3. J. Bacteriol. 194, 5974-5975. doi: 10.1128/JB.01430-12

Man, S., Cheng, R., Miao, C., Gong, Q., Gu, Y., Lu, X., et al. (2011). Artificial trans-encoded small non-coding RNAs specifically silence the selected gene expression in bacteria. Nucleic Acids Res. 39:e50. doi: 10.1093/nar/ gkr034

Marchais, A., Naville, M., Bohn, C., Bouloc, P., and Gautheret, D. (2009). Single-pass classification of all noncoding sequences in a bacterial genome using phylogenetic profiles. Genome Res. 19, 1084-1092. doi: 10.1101/gr.089 714.108

Masse, E., and Gottesman, S. (2002). A small RNA regulates the expression of genes involved in iron metabolism in Escherichia coli. Proc. Natl. Acad. Sci. U.S.A. 99:4620-4625. doi: 10.1073/pnas.032066599

McHugh, C. A., Russell, P., and Guttman, M. (2014). Methods for comprehensive experimental identification of RNA-protein interactions. Genome Biol. 15, 203. doi: $10.1186 / \mathrm{gb} 4152$

Michaux, C., Verneuil, N., Hartke, A., and Giard, J. C. (2014). Physiological roles of small RNA molecules. Microbiology 160(Pt 6), 1007-1019. doi: 10.1099/mic. 0.076208-0

Modi, S. R., Camacho, D. M., Kohanski, M. A., Walker, G. C., and Collins, J. J. (2011). Functional characterization of bacterial sRNAs using a network biology approach. Proc. Natl. Acad. Sci. U.S.A. 108, 15522-15527. doi: 10.1073/pnas. 1104318108

Møller, T., Franch, T., Højrup, P., Keene, D. R., Bächinger, H. P., Brennan, R. G., et al. (2002). Hfq: a bacterial Sm-like protein that mediates RNA-RNA interaction. Mol. Cell 9, 23-30. doi: 10.1016/s1097-2765(01)004361

Muro-Pastor, A. M. (2014). The heterocyst-specific NsiR1 small RNA is an early marker of cell differentiation in cyanobacterial filaments. MBio 5:e01079-14. doi: $10.1128 / \mathrm{mBio} .01079-14$

Naville, M., and Gautheret, D. (2010). Premature terminator analysis sheds light on a hidden world of bacterial transcriptional attenuation. Genome Biol. 11:R97. doi: 10.1186/gb-2010-11-9-r97

Nawrocki, E. P., and Eddy, S. R. (2007). Query-dependent banding (QDB) for faster RNA similarity searches. PLoS Comput. Biol. 3:e56. doi: 10.1371/journal.pcbi. 0030056

Nord, S., Bylund, G. O., Lovgren, J. M., and Wikstrom, P. M. (2009). The RimP protein is important for maturation of the $30 \mathrm{~S}$ ribosomal subunit. J. Mol. Biol. 386, 742-753. doi: 10.1016/j.jmb.2008.12.076

Ott, A., Idali, A., Marchais, A., and Gautheret, D. (2012). NAPP: the nucleic acid phylogenetic profile database. Nucleic Acids Res. 40, D205-D209. doi: 10.1093/ nar/gkr807

Pagel, M., and Johnstone, R. A. (1992). Variation across species in the size of the nuclear genome supports the junk-DNA explanation for the C-value paradox. Proc. Biol. Sci. 249, 119-124. doi: 10.1098/rspb.1992.0093

Papenfort, K., Forstner, K. U., Cong, J. P., Sharma, C. M., and Bassler, B. L. (2015). Differential RNA-seq of Vibrio cholerae identifies the VqmR small RNA as a regulator of biofilm formation. Proc. Natl. Acad. Sci. U.S.A. 112, E766-E775. doi: $10.1073 /$ pnas. 1500203112

Pichon, C., and Felden, B. (2005). Small RNA genes expressed from Staphylococcus aureus genomic and pathogenicity islands with specific expression among pathogenic strains. Proc. Natl. Acad. Sci. U.S.A. 102, 14249-14254. doi: 10.1073/ pnas. 0503838102

Ramos, C. G., da Costa, P. J., Doring, G., and Leitao, J. H. (2012). The novel cis-encoded small RNA h2cR is a negative regulator of hfq2 in Burkholderia cenocepacia. PLoS ONE 7:e47896. doi: 10.1371/journal.pone.0047896

Rivas, E., and Eddy, S. R. (2001). Noncoding RNA gene detection using comparative sequence analysis. BMC Bioinformatics 2:8. doi: 10.1186/14712105-2-8
Romeo, T. (1998). Global regulation by the small RNA-binding protein CsrA and the non-coding RNA molecule CsrB. Mol. Microbiol. 29, 1321-1330. doi: $10.1046 / j .1365-2958.1998 .01021 . x$

Salvail, H., and Masse, E. (2012). Regulating iron storage and metabolism with RNA: an overview of posttranscriptional controls of intracellular iron homeostasis. Wiley Interdiscip. Rev. RNA 3, 26-36. doi: 10.1002/wrna.102

Schmidtke, C., Findeiss, S., Sharma, C. M., Kuhfuss, J., Hoffmann, S., Vogel, J., et al. (2012). Genome-wide transcriptome analysis of the plant pathogen Xanthomonas identifies sRNAs with putative virulence functions. Nucleic Acids Res. 40, 2020-2031. doi: 10.1093/nar/gkr904

Silvaggi, J. M., Perkins, J. B., and Losick, R. (2006). Genes for small, noncoding RNAs under sporulation control in Bacillus subtilis. J. Bacteriol. 188, 532-541. doi: 10.1128/JB.188.2.532-541.2006

Sittka, A., Lucchini, S., Papenfort, K., Sharma, C. M., Rolle, K., Binnewies, T. T., et al. (2008). Deep sequencing analysis of small noncoding RNA and mRNA targets of the global post-transcriptional regulator, Hfq. PLoS Genet. 4:e1000163. doi: 10.1371/journal.pgen.1000163

Soutourina, O. A., Monot, M., Boudry, P., Saujet, L., Pichon, C., Sismeiro, O., et al. (2013). Genome-wide identification of regulatory RNAs in the human pathogen Clostridium difficile. PLoS Genet. 9:e1003493. doi: 10.1371/journal. pgen. 1003493

Stubben, C. J., Micheva-Viteva, S. N., Shou, Y., Buddenborg, S. K., Dunbar, J. M., and Hong-Geller, E. (2014). Differential expression of small RNAs from Burkholderia thailandensis in response to varying environmental and stress conditions. BMC Genomics 15:385. doi: 10.1186/1471-2164-15-385

Thomason, M. K., Fontaine, F., De Lay, N., and Storz, G. (2012). A small RNA that regulates motility and biofilm formation in response to changes in nutrient availability in Escherichia coli. Mol. Microbiol. 84, 17-35. doi: 10.1111/j.13652958.2012.07965.x

Thomason, M. K., and Storz, G. (2010). Bacterial antisense RNAs: how many are there, and what are they doing? Annu. Rev. Genet. 44, 167-188. doi: 10.1146/ annurev-genet-102209-163523

Thompson, J. D., Higgins, D. G., and Gibson, T. J. (1994). CLUSTAL W: improving the sensitivity of progressive multiple sequence alignment through sequence weighting, position-specific gap penalties and weight matrix choice. Nucleic Acids Res. 22, 4673-4680. doi: 10.1093/nar/22.22.4673

Tiedje, J. M. (2002). Shewanella-the environmentally versatile genome. Nat. Biotechnol. 20, 1093-1094. doi: 10.1038/nbt1102-1093

Toledo-Arana, A., Dussurget, O., Nikitas, G., Sesto, N., Guet-Revillet, H., Balestrino, D., et al. (2009). The Listeria transcriptional landscape from saprophytism to virulence. Nature 459, 950-956. doi: 10.1038/nature08080

Vezzi, A., Campanaro, S., D’angelo, M., Simonato, F., Vitulo, N., Lauro, F., et al. (2005). Life at depth: Photobacterium profundum genome sequence and expression analysis. Science 307, 1459-1461. doi: 10.1126/science.1103341

Vogel, J., Gerhart, E., and Wagner, H. (2005). "Approaches to identify novel nonmessenger RNAs in bacteria and to investigate their biological functions: RNA mining," in Handbook of RNA Biochemistry, eds R. K. Hartmann, A. Bindereif, A. Schön, and E. Westhof (Weinheim: Wiley-VCH Verlag GmbH), 595-613. doi: $10.1002 / 9783527619504 . c h 36$

Vogel, J., and Papenfort, K. (2006). Small non-coding RNAs and the bacterial outer membrane. Curr. Opin. Microbiol. 9, 605-611. doi: 10.1016/j.mib.2006.10.006

Vogel, J., and Sharma, C. M. (2005). How to find small non-coding RNAs in bacteria. Biol. Chem. 386, 1219-1238. doi: 10.1515/BC.2005.140

Vogel, J., and Wagner, E. G. (2007). Target identification of small noncoding RNAs in bacteria. Curr. Opin. Microbiol. 10, 262-270. doi: 10.1016/j.mib.2007.06.001

Voss, B., Georg, J., Schon, V., Ude, S., and Hess, W. R. (2009). Biocomputational prediction of non-coding RNAs in model cyanobacteria. BMC Genomics 10:123. doi: 10.1186/1471-2164-10-123

Wagner, E., Altuvia, S., and Romby, P. (2002). 12 Antisense RNAs in bacteria and their genetic elements. Adv. Genet. 46, 361-398. doi: 10.1016/s0065-2660(02) 46013-0

Wang, F., Wang, J., Jian, H., Zhang, B., Li, S., Wang, F., et al. (2008). Environmental adaptation: genomic analysis of the piezotolerant and psychrotolerant deepsea iron reducing bacterium Shewanella piezotolerans WP3. PLoS ONE 3:e1937. doi: 10.1371/journal.pone.0001937

Wang, F., Wang, P., Chen, M., and Xiao, X. (2004). Isolation of extremophiles with the detection and retrieval of Shewanella strains in deep-sea sediments from the west Pacific. Extremophiles 8, 165-168. doi: 10.1007/s00792-003-0365-0 
Washietl, S., and Hofacker, I. L. (2004). Consensus folding of aligned sequences as a new measure for the detection of functional RNAs by comparative genomics. J. Mol. Biol. 342, 19-30. doi: 10.1016/j.jmb.2004.07.018

Washietl, S., Hofacker, I. L., and Stadler, P. F. (2005). Fast and reliable prediction of noncoding RNAs. Proc. Natl. Acad. Sci. U.S.A. 102, 2454-2459. doi: 10.1073/ pnas.0409169102

Wassarman, K. M., Repoila, F., Rosenow, C., Storz, G., and Gottesman, S. (2001). Identification of novel small RNAs using comparative genomics and microarrays. Genes Dev. 15, 1637-1651. doi: 10.1101/gad.901001

Waters, L. S., and Storz, G. (2009). Regulatory RNAs in bacteria. Cell 136, 615-628. doi: 10.1016/j.cell.2009.01.043

Webster, R. E., and Gross, S. R. (1965). The $\alpha$-isopropylmalate synthetase of Neurospora. I. The kinetics and end product control of $\alpha$-isopropylmalate synthetase function*. Biochemistry 4, 2309-2318. doi: 10.1021/bi00887a008

Weinberg, Z., Wang, J. X., Bogue, J., Yang, J., Corbino, K., Moy, R. H., et al. (2010). Comparative genomics reveals 104 candidate structured RNAs from bacteria, archaea, and their metagenomes. Genome Biol. 11:R31. doi: 10.1186/gb-201011-3-r31

Wilms, I., Overloper, A., Nowrousian, M., Sharma, C. M., and Narberhaus, F. (2012). Deep sequencing uncovers numerous small RNAs on all four replicons of the plant pathogen Agrobacterium tumefaciens. RNA Biol. 9, 446-457. doi: $10.4161 /$ rna.17212
Xiao, X., Wang, P., Zeng, X., Bartlett, D. H., and Wang, F. (2007). Shewanella psychrophila sp. nov. and Shewanella piezotolerans sp. nov., isolated from west Pacific deep-sea sediment. Int. J. Syst. Evol. Microbiol. 57(Pt 1), 60-65. doi: 10.1099/ijs.0.64500-0

$\mathrm{Xu}, \mathrm{X}$., Ji, Y., and Stormo, G. D. (2009). Discovering cis-regulatory RNAs in Shewanella genomes by support vector machines. PLoS Comput. Biol. 5:e1000338. doi: 10.1371/journal.pcbi.1000338

Yang, X. W., He, Y., Xu, J., Xiao, X., and Wang, F. P. (2013). The regulatory role of ferric uptake regulator (Fur) during anaerobic respiration of Shewanella piezotolerans WP3. PLoS ONE 8:e75588. doi: 10.1371/journal.pone.00 75588

Conflict of Interest Statement: The authors declare that the research was conducted in the absence of any commercial or financial relationships that could be construed as a potential conflict of interest.

Copyright (c) 2017 Nawaz, Jian, He, Xiong, Xiao and Wang. This is an open-access article distributed under the terms of the Creative Commons Attribution License (CC BY). The use, distribution or reproduction in other forums is permitted, provided the original author(s) or licensor are credited and that the original publication in this journal is cited, in accordance with accepted academic practice. No use, distribution or reproduction is permitted which does not comply with these terms. 